\title{
Nonperturbative Quark-Gluon Thermodynamics at Finite Density
}

\author{
Maxim Andreichikov * \\ Institute for Theoretical and Experimental Physics \\ E-mail: andreichicov@mail.ru

\section{Yuri Simonov} \\ Institute for Theoretical and Experimental Physics \\ E-mail: simonoveitep.ru
}

\begin{abstract}
Thermodynamics of the quark-gluon plasma at finite density is studied with the vacuum correlator method. The contribution of the nonperturbative effects is defined by the Polyakov loops and colormagnetic confinement. An analytical expression was obtained for the equation of state $P(T, \mu)$ for $0<\mu<400 \mathrm{MeV}$ in a good agreement with current lattice data at zero density. A set of branching points were found for the $P(T, \mu)$ in a complex $\mu$-plane which coincide with the Roberge-Weiss singularities.
\end{abstract}

XIII Quark Confinement and the Hadron Spectrum - Confinement2018

31 July - 6 August 2018

Maynooth University, Ireland

\footnotetext{
*Speaker.
} 


\section{Introduction}

The new phenomenon of the quark-gluon plasma (QGP) was predicted in [1], and its properties were soon measured on the lattice [2]. Nowadays the QGP is an important stage in the heavy-ion collisions is widely recognized as see e.g. [3].

Recent accurate lattice calculations [4, 5] show that the QGP demonstrates non-trivial behavior due to the strong nonperturbative (np) interaction during the phase transition and beyond it. Field Correlator Method (FCM) was originally formulated in [6] makes it possible to include np effects in a consistent way with so-called vacuum field correlators. The deconfining phase transition in FCM approach is associated with the vanishing of the color-electric (CE) correlator $D^{E}$, and an another $n p$ correlator $D_{1}^{E}$ provides the Polyakov loop potential [7]. Recently another important ingredient of the $n p$ interaction in the region $T>T_{c}$ was taken into account - the color-magnetic (CM) confinement which is defined by the spatial projection of the Wilson loop and by the $n p$ correlator $D^{H}$. In $[8,9,10]$ this theory was fully investigated in the case of SU(3) and zero-density compared to the accurate lattice data $[4,10]$, showing a good agreement for $p(T)$ both below and above $T_{c}$, and also in the character of transition. Meanwhile the region of nonzero density is of the outmost importance. The existing and planned heavy ion collision experiments badly need the corresponding theoretical calculations of the QGP properties at nonzero $\mu$, whereas the lattice data are not directly available in this region at the moment due to sign problem. FCM approach to the case of the finite density was formulated in [11], see also [10, 12] for a review, where in the QGP only the Polyakov line interaction was taken into account, and the whole temperature transition curve in the $\mu-T$ plane was found.

It is the purpose of the present paper to incorporate in our calculations of $n p$ thermodynamics for finite density the effects $\mathrm{CM}$ confinement and produce the function of pressure $p(T, \mu)$, in the temperature interval $0.2<T<1.0 \mathrm{GeV}$. It is also interesting to investigate the properties of $p(T, \mu)$ in the whole complex plane of $\mu$ and to compare with the known lattice information.

\section{Equation of state for the quark-gluon plasma}

We are using below the same gauge and relativistic invariant formalism, based on the path integral formalism, which was formulated in [6, 7], and exploited in the $S U(3)$ case in [13]. The basic interaction of a quark or a gluon can be expressed via world lines affected by the vacuum fields and finally written in the form of Wilson-lines and loops averaged over the background field.

As was mentioned in the Introduction, two basic interactions define the quark and gluon dynamics in QGP beyond the phase transition: the colorelectric (CE) one, contained in the Polyakov line $L(T)$, and the colormagnetic $(\mathrm{CM})$ interaction in the spatial projection on the Wilson loop. The CE part is expressed via the $n p$ part of the CE field correlator $D_{1}^{E}(\tau)$, while perturbative part of $D_{1}$ yields color Coulomb potential. The CM part is defined by the CM field correlator $D^{H}(z)$, yielding the spatial string tension $\sigma_{s}(T)=\frac{1}{2} \int D^{H}(z) d^{2} z$. It was shown in [14] that $\sigma_{s}(T) \sim g^{2}(T) T^{2}$ and is important in the whole region $T \geq T_{c}$.

Using the path integral formalism with periodic boundary conditions, one can express the thermodynamic potentials via the Wilson loop integral, e.g. for the gluon pressure one has [14, 9] 


$$
P_{g l}=2\left(N_{c}^{2}-1\right) \int_{0}^{\infty} \frac{d s}{s} \sum_{n=1,2 \ldots} G^{(n)}(s)
$$

where $G^{(n)}(s)$ is the winding (Matsubara) path integral in Feynman-Fock-Schwinger representation

$$
G^{(n)}(s)=\int(D z)_{o n}^{w} e^{-K}\left\langle\hat{t}_{a} W\left(C_{n}\right)\right\rangle .
$$

Here $W\left(C_{n}\right)$ is the Wilson loop defined for the gluon path $C_{n}$, which has both temporal (i4) and spatial projections (ij). It is important, that the $\mathrm{CE}$ and $\mathrm{CM}$ field strengths correlate very weakly $\left\langle E_{i}(x) B_{k}(y) \Phi(x, y)\right\rangle \approx 0[7]$ and therefore one can factorize CE and CM interactions in the $W\left(C_{n}\right)$ [9]

$$
\left\langle\operatorname{tr}_{a} W\left(C_{n}\right)\right\rangle=L_{\text {adj }}^{(n)}(T)\left\langle W_{3}\right\rangle .
$$

Statement (2.3) leads to the same factorization for the Green's function $G^{(n)}(s)$

$$
G^{(n)}(s)=G_{4}^{(n)}(s) G_{3}(s) ; G_{4}^{(n)}(s)=\int(D z)_{o n}^{w} e^{-K} L_{n}^{(n)}=\frac{1}{2 \sqrt{4 \pi s}} e^{-n^{2} / 4 T^{2} s} L^{(n)} .
$$

where the temporal projection $G_{4}^{(n)}(s)$ is defined by the Polyakov loop interaction $L^{(n)}$. As shown in [7, 12], $L_{\text {adj }}^{(n)} \cong\left(L_{\text {adj }}(T)\right)^{n}$ for $T \lesssim 1 \mathrm{GeV}$, and $L_{\text {adj }}(T)=\exp \left(-\frac{9 V_{1}(\infty, T)}{8 T}\right)$. According tp [9], then the resulting $L_{\text {adj }}(T)$, which is close to the lattice measurement values, yields realistic thermodynamic potentials. As a result, one can write the resulting expression for the gluon pressure

$$
P_{g l}=\frac{N_{c}^{2}-1}{\sqrt{4 \pi}} \int_{0}^{\infty} \frac{d s}{s^{3 / 2}} G_{3}(s) \sum_{n=0,1,2 \ldots} e^{-\frac{n^{2}}{4 T^{2} s}} L_{\mathrm{adj}}^{(n)} .
$$

In case of the linear CM confinement, the spatial projection $G_{3}(s)$ could be calculated analytically [9]

$$
G_{3}^{l i n}(s)=\frac{1}{(4 \pi s)^{3 / 2}} \sqrt{\frac{\left(M_{\mathrm{adj}}^{2} s\right)}{\sinh \left(M_{\mathrm{adj}}^{2} s\right)}},
$$

where $M_{\text {adj }} \cong 2 M_{D} \sim 4 \sqrt{\sigma_{s}}$ is defined by the spatial string tension and is proportional to the gluon Debye mass $M_{D}$. Spatial string tension grows rapidly with temperature, i.e. $\sigma_{s} \sim g^{2}(T) T^{2}$, providing an effective damping for the pressure. Due to this effect, the QGP pressure never reaches the Stefan-Boltzmann limit. This behavior is in agreement with the current lattice data from [15].

The same FCM technique could be reproduced for quarks in fundamental representation following $[7,11]$, but augmented by the quark mass term $e^{-m_{f}^{2} s}$ and the density term $\cosh \frac{\mu n}{T}$.

$$
P_{q}=\sum_{m_{q}} P_{q}^{(f)}, P_{q}^{(f)}=\frac{4 N_{c}}{\sqrt{4 \pi}} \int_{0}^{\infty} \frac{d s}{s^{3 / 2}} e^{-m_{f}^{2} s} S_{3}(s) \sum_{n=1,2, . .}(-)^{n+1} e^{-\frac{n^{2}}{4 T^{2} s}} \cosh \left(\frac{\mu n}{T}\right) L_{f}^{n} .
$$

Here $S_{3}(s)$ is, similarly to $G_{3}(s)$, the 3 d quark Green's function, which has the same form as (2.6) with the relation $M_{\text {adj }}^{2}=\frac{9}{4} M_{f}^{2}$. 


\section{Properties of the equation of state at finite density}

The full pressure for the QGP is $P(T, \mu)=P_{g l}(T)+P_{q}(T, \mu)$, where $P_{q}(T, \mu)$ is given in (2.7). In previous Section we have used the linear potential for the $\mathrm{CM}$ confinement to obtain the spectral representation of the spatial Green's function (2.6). In the case of the arbitrary CM interaction one has the most general spectral representation for $S_{3}(s)$ is [9]

$$
S_{3}(s)=\frac{1}{\sqrt{\pi s}} \sum_{v=0,1} \psi_{v}^{2}(0) e^{-m_{v}^{2} s}
$$

with the corresponding eigenvalues $m_{v}$ and wave functions $\psi_{v}$. The $P_{q}^{(f)}$ acquire the form

$$
\frac{P_{q}^{(f)}}{T^{4}}=\frac{8 N_{c}}{\pi T^{3}} \sum_{v} \psi_{v}^{2}(0) \bar{M}_{v} \sum_{n} \frac{(-)^{n+1}}{n} K_{1}\left(\frac{n \bar{M}_{v}}{T}\right) L^{n} \cosh \frac{\mu n}{T},
$$

where the damping masses are $\bar{M}_{v}^{2}=m_{f}^{2}+m_{v}^{2}$. Summing over the Matsubara frequences one can write down the result

$$
\frac{P_{q}^{(f)}}{T^{4}}=\frac{8 N_{c}}{\pi T^{4}} \sum_{v=0}^{\infty} \psi_{v}^{2}(0) \bar{M}_{v}^{2} \int_{0}^{\infty} \sinh ^{2} t d t \frac{1}{2}\left(\frac{\alpha_{+}}{1+\alpha_{+}}+\frac{\alpha_{-}}{1+\alpha_{-}}\right),
$$

with $\alpha_{ \pm}=\exp \left\{-\frac{\bar{M}_{v}}{T} \cosh t-\frac{V_{1}}{2 T} \pm \frac{\mu}{T}\right\}$. One can see that there is a set of singularities for the pressure in a complex $\mu$-plane, shown in Fig. 1. To this end we are writing $\frac{\mu}{T}$ in the neighborhood of the point in Fig. 1 with imaginary and real parts $i \pi$ and $\frac{\mu_{R}}{T}$ respectively as

$$
\frac{\mu}{T}=i \pi+\frac{M_{v}+V_{1} / 2}{T}+\frac{M_{v}}{T} y
$$

Note, that for the prefactor of the exponent $\alpha_{ \pm}$in (3.3) be equal to $(-1)$ the imaginary part of $\mu$ should equal

$$
\frac{\mu_{I}}{T}=\frac{\pi}{3}(2 n+1), \quad n=0, \pm 1, \pm 2, \ldots
$$

This situation may explain the appearance of the Roberge-Weiss singularities [16], see [17], [18] for a physical and numerical analysis. The integral (3.3) as a function of $y$ is proportional to the function $f(y)$,

$$
f(y)=\int_{0}^{\infty} \frac{t^{2} d t F(t)}{t^{2}-2 y+O\left(t^{2} y, t^{4} y^{2}\right)},
$$

where we have separated the region of small $t$, contributing to the singularity, and $F(t \rightarrow 0)=$ const. One can easily see in (3.6), that $f(y)$ has a square root singularity near $y=0$ and the cut $\operatorname{Re} y \geq 0$, with the discontinuity

$$
f(y+i \delta)-f(y-i \delta)=i \sqrt{2 y} F(\sqrt{2 y}) .
$$

Note, that the branch points $y=0$ occur for every $M_{v}, v=0,1,2, \ldots$ and this situation is similar to the two-body thresholds in the energy plane with ever increasing number of particles. 


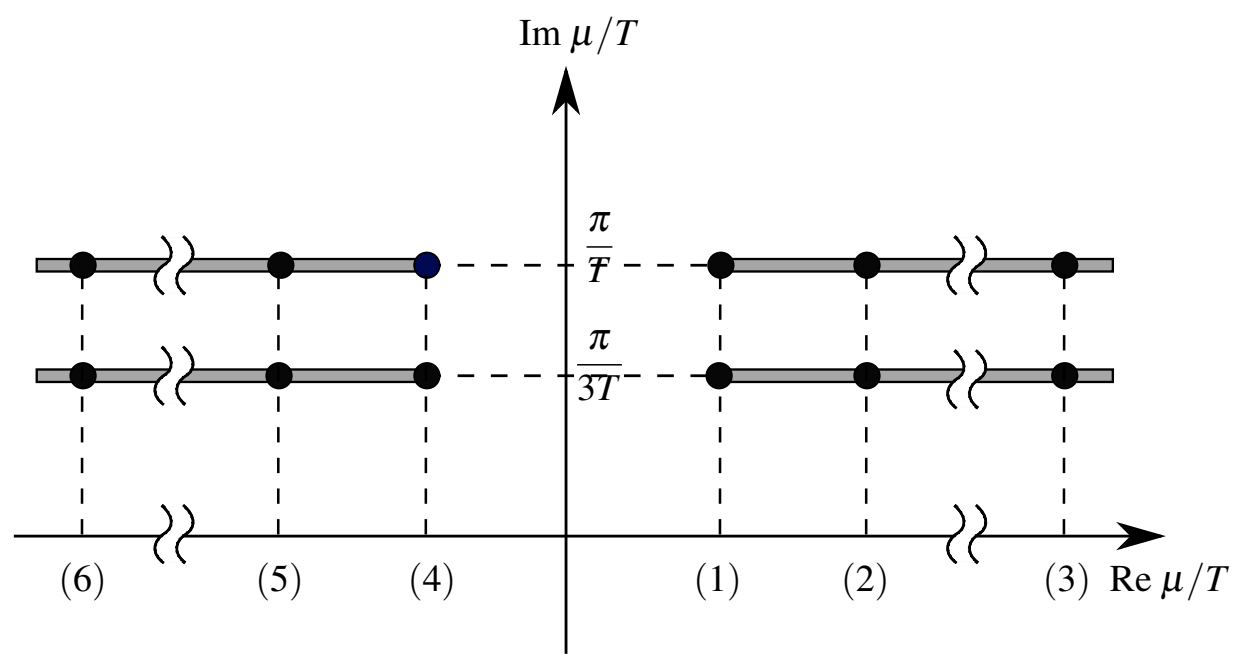

Figure 1: Roberge-Weiss singular points and cuts in the complex plane of $\mu$. Points 1-6 are, respectively, $\left(\frac{V_{1}}{2 T}+\frac{M_{0}}{T}\right),\left(\frac{V_{1}}{2 T}+\frac{M_{1}}{T}\right),\left(\frac{V_{1}}{2 T}+\frac{M_{V}}{T}\right),-\left(\frac{V_{1}}{2 T}+\frac{M_{0}}{T}\right),-\left(\frac{V_{1}}{2 T}+\frac{M_{1}}{T}\right)$, and $-\left(\frac{V_{1}}{2 T}+\frac{M_{V}}{T}\right)$. In the lower half plane the points are mirror-reflected of the axis $\operatorname{Re} \mu / T$.

\section{Results and discussion}

In this section we present results of calculations for the total pressure

$$
P(\mu, T)=P_{g l}(T)+\sum_{m_{q}(i)} P_{q}^{(f)}\left(\mu_{i}, T\right)
$$

where $P\left(\mu_{f}, T\right)$ in general depends on the $\mu_{f}$ for a given flavor. Below we consider the simplest case of equal $\mu_{f}=\mu$, where $f=u, d, s$, and the quark masses $m_{u}=m_{d}=0, m_{s}=0.1 \mathrm{GeV}$. $P_{g l}(T)$ is given in Eq.(2.5) and is $\mu$-independent in our approximation of no interaction between quarks and gluons. We used the values $\psi_{v}$ and $M_{v}$ corresponding to the linear CM interaction to calculate the $P_{f}(T, \mu)$. The resulting behavior of the $P(T, \mu) / T^{4}$ is shown in Fig. 2 for $\mu=0,0.2,0.4$ $\mathrm{GeV}$, where also the case of $\mu=0$ can be compared to lattice data [21]. One can see a reasonable agreement with lattice data for $\mu=0$. Note, that for the Polyakov loop $L_{f}(T)=\exp \left(-V_{1}(T) / 2 T\right)$ we are using as in Eq. (2.5) and Eq. (2.7) the same values as in [13, 11, 12], with $L_{a d j}=\left(L_{f}\right)^{9 / 4}$, namely

$$
V_{1}(T)=\frac{0.175 \mathrm{GeV}}{1.35 \frac{T}{T_{0}}-1}, \quad T_{0}=0.16 \mathrm{GeV}
$$

in the interval $0.16 \mathrm{GeV}<T<1 \mathrm{GeV}$. The resulting values of $L_{f}(T)$ are in the same domain as the lattice data of [22] for $T<0.5 \mathrm{GeV}$, while at higher $T$ our $L_{f}$ is smaller due to necessary renormalization, because of different definitions, see [23] for the discussion of this point. Also there is a possibility of the $\mu$-dependence of $L_{f}(T)$, which can occur due to density dependence of vacuum fields, as well as due to quark-quark interaction. In our approach at this stage we disregard this dependence, which is partly supported by lattice data [24]. 


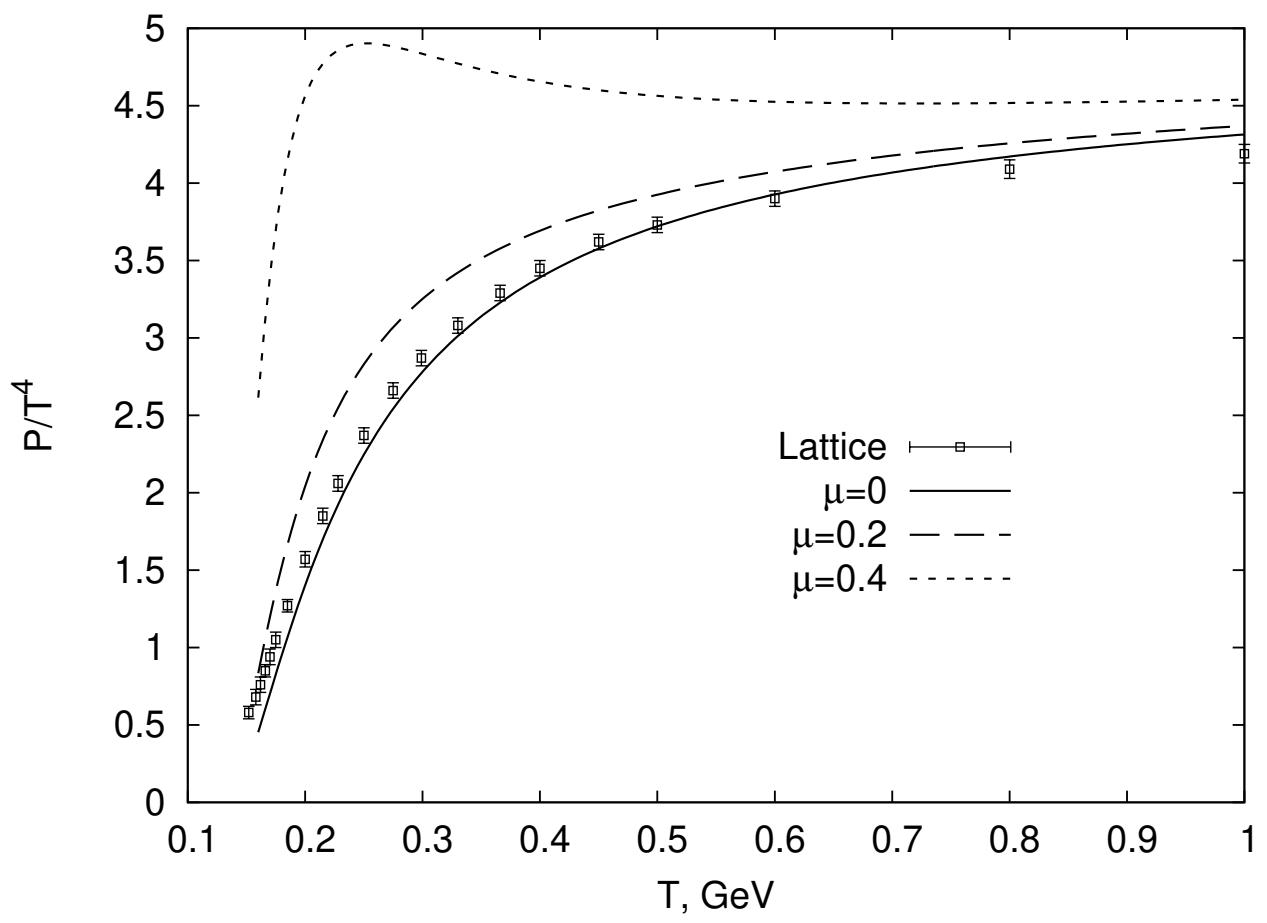

Figure 2: The pressure $\frac{P(T)}{T^{4}}$ with $M_{0}=3.5 \sqrt{\sigma_{s}}$ for $\mu=0.0,0.2,0.4$ (from bottom to top), empty squares with error bars are for the lattice data from [21].

We have considered above the propeties of the QGP in the temperature interval $0.15 \mathrm{GeV}<$ $T<1 \mathrm{GeV}$ and for the chemical potential $\mu=0,0.2$ and $0.4 \mathrm{GeV}$. We have taken into account only the $n p$ part of interaction. The main reason for our choice of dynamics is the fact, that the CM confinement and Polyakov interaction $\left(V_{1}(T)\right)$ are most strong in this region and moreover CM confinement is growing with temperature.

We have analyzed the behavior of $P(T, \mu)$ up to $\mu=0.4 \mathrm{GeV}$ and have not found any discontinuous effects in this area. It is seen in Fig. 2 that the pressure has a smooth behavior, while the peak in $P(T, \mu)$ appears at smaller $T$ with increasing $\mu$. It was checked, that the series over $n$ in Eq. (3.2) is convergent for these values of $\mu$. At the same time we have studied above the analytic properties of thermodynamic potentials in the complex $\mu$ plane and have found sequences of branch points with cuts, going outwards, see Fig. 1. These singularities and cuts are dynamically explained by the Polyakov line interaction $V_{1}(T)$ and CM confinement eigenvalues $M_{v}$.

This work was done in the framework of the scientific project, supported by the Russian Scientific Foundation, grant \#16-12-10414.

\section{References}

[1] J. C. Collins, M. J. Perry, Phys. Rev. Lett. 34, 1353 (1975). N. Cabibbo, G. Parisi, Phys. Lett. B 59, 67 (1975). E. V. Shuryak, Sov. Phys. JETP 47, 212 (1978).

[2] M. Creutz, Phys. Rev. D 21, 2308 (1980).L. D. McLerran, B. Svetitsky, Phys. Lett. B 98, 195 (1981).J. Kuti, J. Polonyi, K. Szlachanyi, Phys. Lett. B 98, 199 (1981). 
[3] P. Braun-Munzinger, V. Koch, Th. Schäfer and J. Stachel, Phys. Rept. 621, 76 (2016) [arXiv:1510.00442 [nucl-th]]. A. Andronic, P. Braun-Munzinger, K. Redlich and J. Stachel, J. Phys.: Conf. Ser. 779, 012012 (2017) [arXiv:1611.01347 [nucl-th]]. M. Albright, J. Kapusta and C. Young, Phys. Rev. C 90, 024915 (2014) [arXiv:1404.7540 [nucl-th]]. J. Cleymans, H. Oeschler, K. Redlich and S. Wheaton, Phys. Rev. C 73, 034905 (2006) [arXiv:hep-ph/0511094].

[4] Sz. Borsanyi, G. Endrödi, Z. Fodor, A.D. Katz and K.K. Szabo, JHEP, 2012(07): 056 (2012), [arXiv:1204.6184 [hep-lat]].

[5] L. Giusti and M. Pepe, Phys. Lett. B 769, 385 (2017) [arXiv:1612.00265 [hep-lat]]. L. Giusti and M. Pepe, PoS (LATTICE-2016): 061 [arXiv:1612.02337 [hep-lat]].

[6] Yu.A. Simonov, JETP Lett. 54, 249 (1991). Yu.A. Simonov, JETP Lett. 55, 627 (1992).

Yu.A. Simonov, Phys. At. Nucl. 58, 309 (1995) [hep-ph/9311216]. N.O. Agasian, JETP Lett. 57, 208 (1993). N.O. Agasian, JETP Lett. 71, 43 (2000). H.G. Dosch, H.J. Pirner, Yu.A. Simonov, Phys. Lett. B 349335 (1995). Yu.A. Simonov, in “Varenna-1995: Selected Topics in Nonperturbative QCD”, Eds. A.Di Giacomo and D. Diakonov (IOS Press, Italy, 1996), p. 319 [hep-ph/9509404].

[7] Yu.A. Simonov, Ann. Phys. 323, 783 (2008) [arXiv: hep-ph/0702266]. E.V. Komarov, Yu.A. Simonov, Ann. Phys. 323, 1230 (2008) [arXiv:0707.0781 [hep-ph]].

[8] N.O. Agasian, M.S. Lukashov and Yu.A. Simonov, Mod. Phys. Lett. A 31, 1050222 (2016) [arXiv:1610.01472 [hep-lat]].

[9] N.O. Agasian, M.S. Lukashov and Yu.A. Simonov, Eur. Phys. J. A 53: 138 (2017) [arXiv:1701.07959 [hep-ph]].

[10] M.A. Andreichikov, M.S. Lukashov, Yu.A. Simonov, Int. J. Mod. Phys. A33, 8, 1850043 (2018) [arXiv:1707.04631 [hep-ph]].

[11] Yu.A. Simonov, M.A. Trusov, JETP Lett. 85, 598 (2007) [arXiv:hep-ph/0703228]. Yu.A. Simonov, M.A. Trusov, Phys. Lett. B 650, 36 (2007) [arXiv:hep-ph/0703277].

[12] A.V. Nefediev, Yu.A. Simonov and M.A. Trusov, Int. J. Mod. Phys. E 18, 549 (2009) [ arXiv:0902.0125 [hep-ph]].

[13] M.S. Lukashov and Yu.A. Simonov, JETP Letters 105, 691 (2017) [arXiv: 1703.06666 [hep-ph]].

[14] Yu.A. Simonov, arXiv:1605.07060 [hep-ph].

[15] S.Borsanyi et. al. Phys. Lett. B370 (2014) 99-104, arXiv:1309.5258; JHEP 1011:077, 2010, arXiv:1007.2580.

[16] A. Roberge and N. Weiss, Nucl. Phys. B 275, 734 (1986).

[17] C. Bonati, P. De Forcrand, M. D’Elia, O. Philipsen and F. Sanfilippo, Phys. Rev. D 90, 074030 [arXiv:1408.5086].

[18] R. Falcone, E. Laermann and M.P. Lombardo, PoS (LATTICE-2010): 183 [arXiv:1012.4694 [hep-lat]].

[19] S.A. Gottlieb, W. Lin, D. Toussaint, R.L. Reken and R.L. Sugar, Phys. Rev. Lett. 59, 2247 (1987).

[20] A. Bazavov, H.-T. Ding, P. Hegde, et al., Phys. Rev. D 95, 054504 (2017) [arXiv:1701.04325 [hep-lat]].

[21] Sz. Borsanyi, G. Endrödi, Z. Fodor, et al., JHEP 2010(11): 077 (2010) [arXiv:1007.2580 [hep-lat]]. 
[22] A. Bazavov, N. Brambilla, H.-T. Ding, et al., Phys. Rev. D 93, 114502 (2016) [arXiv:1603.06637 [hep-lat]]. P. Petreczky, H.-P. Schadler, Phys. Rev. D 92, 094517 (2015) [arXiv:1509.07874 [hep-lat]]. O. Kaczmarek, F. Zantow, Phys. Rev. D 71, 114510 (2005) [arXiv:hep-lat/0503017]. S. Borsanyi, S. Durr, Z. Fodor, et al., JHEP 2012(08): 126 (2012) [arXiv:1205.0440 [hep-lat]].

[23] Yu.A. Simonov, Phys. Lett. B 619, 293 (2005) [hep-ph/0502078].

[24] J. Takahashi, K. Nagata, T. Saito, et al., Phys. Rev. D 88, 114504 (2013) [arXiv:1308.2489 [hep-lat]]. 